Neurosurg Focus 17 (1):E5, 2004, Click here to return to Table of Contents

\title{
The Canadian multicenter trial of pallidal deep brain stimulation for cervical dystonia: preliminary results in three patients
}

\author{
Zelma H. T. Kiss, M.D., Ph.D., Kristina Doig, B.N., R.N., Michael Eliasziw, Ph.D., \\ RANJIIT RanaWaYa, M.D., AND OKSANa SuchowersKy, M.D.
}

Departments of Clinical Neuroscience and Community Health Sciences, University of Calgary, Alberta, Canada

\begin{abstract}
Object. Deep brain stimulation (DBS) of the globus pallidus internus (GPi) is beneficial for generalized dystonia and has been proposed as a treatment for cervical dystonia. The Canadian Stereotactic/Functional and Movement Disorders Groups designed a pilot project to investigate the following hypothesis: that bilateral DBS of the GPi will reduce the severity of cervical dystonia at 1 year of follow up, as scored in a blinded fashion by two neurologists using the Toronto Western Spasmodic Torticollis Rating Scale (TWSTRS). Secondary outcome measures included pain and disability subscores of the TWSTRS, Short Form-36 quality of life index, and the Beck Depression Inventory.

Methods. Three patients have undergone surgery in Calgary with a follow-up duration of $7.4 \pm 5.9$ months (mean \pm standard deviation). One patient underwent inadvertent ineffective stimulation for the first 3 months and did not experience a benefit until DBS programming was corrected. All three patients had rapid response to stimulation, with the muscles relaxing immediately and abnormal movements improving within days. Total TWSTRS scores improved by $79 \%$, and severity subscores improved significantly, from $15.7 \pm 2.1$ to $7.7 \pm 2.9$ (paired t-test, $p=0.02$ ). Pain and disability subscores improved from $25.5 \pm 4.1$ to $3.3 \pm 3.1$ (paired t-test, $\mathrm{p}=0.002$ ) and from $13.3 \pm 4.9$ to $3.3 \pm 4.2$ (paired t-test, $\mathrm{p}=0.06$ ), respectively.

Conclusions. Although it is too early to reach broad conclusions, this report of preliminary results confirms the efficacy of DBS of the GPi for cervical dystonia.
\end{abstract}

\section{KEY WoRDS • dystonia • torticollis • globus pallidus • deep brain stimulation • clinical trial}

Cervical dystonia, previously referred to as spasmodic torticollis, is now recognized as a form of focal dystonia. Neck muscle contractions typically begin gradually, occasionally after minor trauma, and progress over years. This results in a variety of abnormal neck movements and postures. ${ }^{19}$ Cervical dystonia may remain mild in some individuals, but in most it becomes severe and disabling with remissions occurring only rarely. ${ }^{18}$ Botulinum toxin injections are the mainstay of treatment, although only $63 \%$ of patients experience long-term sustained benefit. ${ }^{15}$ Thus, a significant proportion are left with disabling torticollis that is not responsive to medical therapy.

Cervical dystonia has been managed using stereotactic surgical procedures since the 1950s; these involve lesioning of a variety of targets ${ }^{3,14,36}$ (see Loher, et al., ${ }^{27}$ for recent review). In addition, deafferenting peripheral nerve $e^{2,24,29}$

Abbreviations used in this paper: BDI = Beck Depression Inventory; DBS = deep brain stimulation; $\mathrm{GPe}=$ globus pallidus externus; $\mathrm{GPi}=$ globus pallidus internus; $\mathrm{IPG}=$ implantable pulse generator; $\mathrm{MR}=$ magnetic resonance; $\mathrm{PD}=$ Parkinson disease $; \mathrm{SF}-$ 36 = Short Form-36; TWSTRS = Toronto Western Spasmodic Torticollis Rating Scale. and intradural root procedure ${ }^{12}$ have also been advocated. Some treatments require multiple surgical procedures or are associated with side effects such as dysphagia and aspiration. ${ }^{12,29}$ The peripheral denervating procedure is performed in a limited number of centers because of the extensive postoperative rehabilitation required. ${ }^{4}$ Because this is a peripheral nerve procedure, the torticollis often recurs as the nerves regenerate ${ }^{29}$ and thus the benefits of surgery wear off over time.

With the resurgence of interest in stereotactic surgery in the 1990s, it was observed that DBS applied in the GPi could eliminate drug-induced dyskinesia and improve dystonia in patients with PD. ${ }^{10}$ This prompted application of DBS to treat generalized dystonia in children ${ }^{7,32}$ and adults, ${ }^{34}$ and excellent results were reported. More recently, the use of pallidal DBS has also been reported in the treatment of adults with refractory cervical dystonia. ${ }^{1,11,17}$, 22,23,26,31 Unfortunately these reports have several limitations: 1) small sample size, whereby seven publications report on a total of 16 patients (some patients are likely to have been reported twice); 2) combining results in focal cervical and generalized dystonias; ;,11,22 3) inconsistent use of objective and validated scales to assess the extent of the 
condition, such as the TWSTRS ${ }^{6}$ or cervical dystonia rating scale; ${ }^{30}$ and 4) in none of the studies were the neurologists performing the clinical assessments working in a blinded fashion. Reports with the aforementioned limitations are common when surgeons are asked to treat the most refractory cases as a last attempt to improve the patient's quality of life and the procedures are never subjected to the scrutiny required of other treatments. Therefore, in 2002 we proposed a Canadian five-center feasibility study to address the shortcomings in the previous publications. Our aim was to determine whether bilateral pallidal DBS results in improvement in neck postures/ movements and quality of life and to document the adverse effects of this treatment in patients with cervical dystonia. In this report, we describe the study design and protocol and focus on the preliminary results-the first three patients treated in the coordinating center. These data have been presented previously in abstract form. ${ }^{20}$

\section{CLINICAL MATERIAL AND METHODS}

All patients who had suffered from refractory and isolated cervical dystonia for at least 5 years and in whom medical management had failed were considered for entry into the study. Only those who consented to undergo DBS surgery with all necessary follow up were enrolled. The primary outcome was the severity subscale of the TWSTRS, as assessed by two neurologists in a blinded fashion. Secondary outcomes included the TWSTRS subscores for pain and disability, the SF-36 score to measure quality of life, results of the BDI, and adverse events. Videotapes and assessments were performed at baseline at least 3 months after the last botulinum toxin injection and at 1 year postoperatively. In addition, swallowing studies and neuropsychological assessments were performed preoperatively and at the 1-year follow-up visit. Patients wore a hospital gown and surgical cap for videotape assessments to maintain blinding for the scoring neurologist. Details of the protocol are shown in Fig. 1.

\section{Surgical Procedure}

Preoperative MR images were used to identify the anterior and posterior commissures and, using the Schaltenbrand and Wahren ${ }^{33}$ brain atlas, an initial target was selected $20 \mathrm{~mm}$ to the right of midline. Microelectrode recordings were then used to map the most posteroventral part of the $\mathrm{GPi},{ }^{28}$ first in the right brain and then in the left. The DBS electrodes (model 3387; Medtronic, Inc., Minneapolis, MN) were then placed with the 0 (lowest) lead at least $2 \mathrm{~mm}$ above the optic tract. At this site the electrodes spanned the entire GPi from ventral to dorsal borders, and the most dorsal DBS leads may have reached the ventral GPe. Electrodes were secured to the calvaria with a silk stitch and methylmethacrylate. Excess DBS wire was coiled in the subperiosteal space and attached to an external lead for postoperative testing. This testing was performed mainly to determine thresholds for side effects and to allow postoperative MR imaging to confirm adequate placement before insertion of the generator.

On Day 3 or 4 after placement of the DBS electrodes, the IPG (Kinetra; Medtronic, Inc.) was placed in a subclavicular pocket on the side to which the patient's neck usually turned. A miniplate was fashioned and secured to the calvaria behind the ear to provide a tunnel under which the extension wires could pass and to prevent dislocation of the connector into the subcutaneous tissue of the neck (Fig. 2). These refinements were introduced to minimize the chance of lead breakage, because significant hardware complications have been described in patients with severe neck movements. ${ }^{38}$ Perioperative antibiotic drugs were administered at the time of surgery and for 24 hours postoperatively.

\section{Programming of the DBS Implant}

Programming was performed by movement disorder nurse clinicians 1 to 2 weeks after implantation. A similar protocol was used as that in patients with $\mathrm{PD}^{16}$ but settings were started at a wider pulse duration (90-210 $\mu$ sec)..$^{22,31}$ Although we initially thought that treatment effects would occur in a delayed manner, ${ }^{7,22}$ this was not the case.

\section{Postoperative Assessments and Follow-Up Review}

Patients were seen by the treating neurologist and neurosurgeon at 3,6 , and 12 months postoperatively, specifically for assessment of adverse events. Adjustments in medications were made by the neurologist and patient as warranted. The TWSTRS disability and pain assessments, severity videotapes, BDI, and SF-36 questionnaire were administered by the nurse coordinator at each site at 6 and 12 months postoperatively. Videotapes and results of these assessments were sent to Calgary at the completion of the 12-month follow-up review. For this preliminary report, the scoring neurologists (O.S. and R.R.) were not blinded to the surgical status of the three patients.

\section{Sample Size}

A sample size of 10 patients was selected because this was deemed to be sufficient to detect a difference in the mean TWSTRS severity score of 4 . This was estimated from published case reports, ${ }^{22,23}$ assuming a baseline severity score of 20 and a standard deviation of differences of 4 . Using a paired t-test at a 5\% two-sided level of significance, a $20 \%$ relative difference would be detected with $80 \%$ statistical power.

\section{Statistical Analysis}

Patients served as their own control; a paired t-test was used to compare outcomes from baseline to last follow-up visit. Results are reported as the mean \pm standard deviation.

\section{SUMMARY OF CASES}

\section{Case 3006}

History. The first patient was a right-handed 63-yearold man with longstanding postural tremor in both hands. ${ }^{13}$ Fourteen years before surgery he had noticed the onset of neck turning toward the left. The most effective sensory trick for relieving the torticollis was touching the side of his face or pushing his head back against a highbacked chair. His neck movements gradually worsened over 5 years, at which time he started receiving injections 


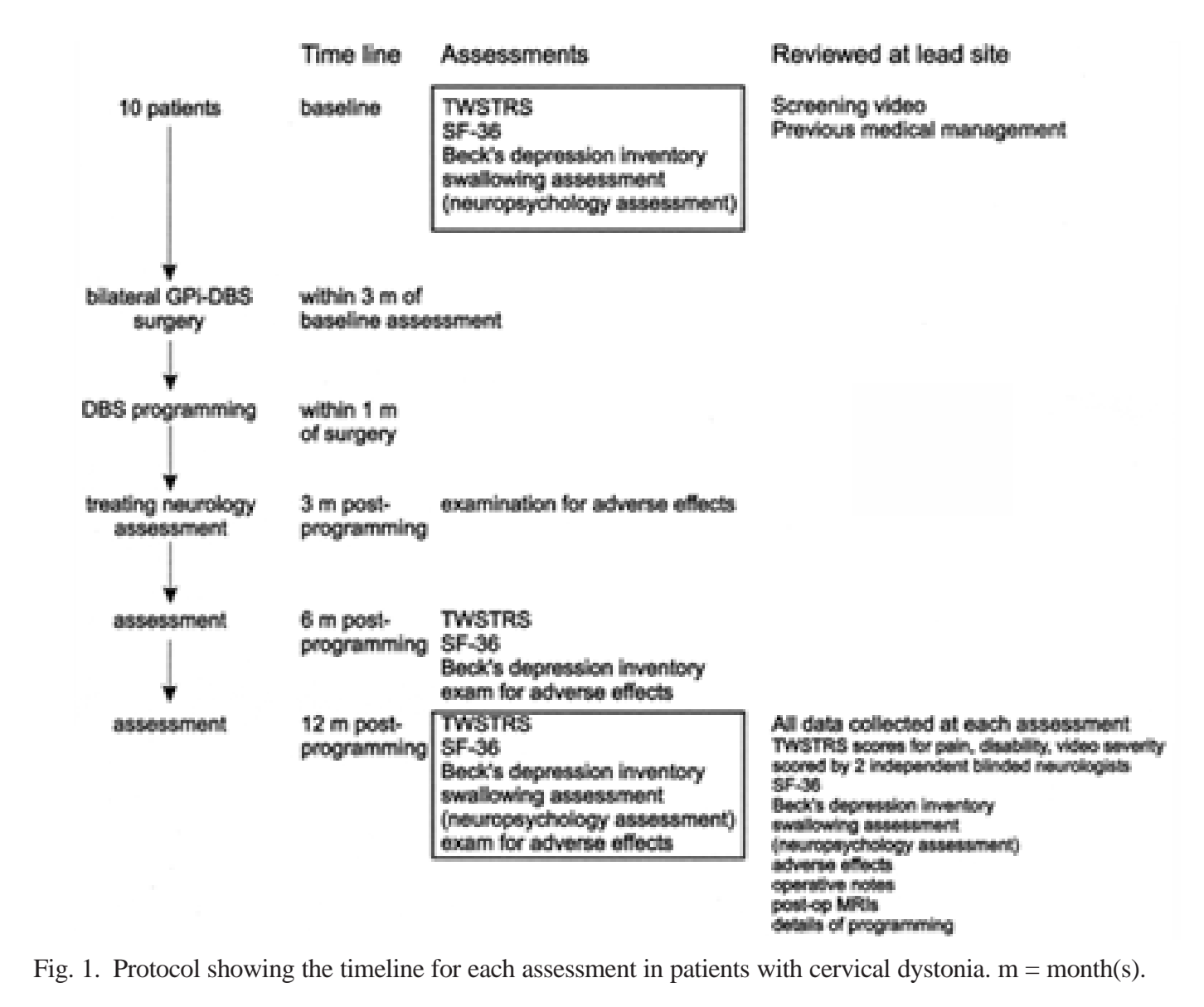

of botulinum toxin and a trial of tetrabenazine. He experienced only minimal improvement after botulinum toxin injections and because pain was a major symptom he underwent C3-4 facet rhizotomy and trials of long-acting narcotic analgesic drugs. Microvascular decompression of the right 11th nerve and upper cervical roots failed to improve his neck movements. He also suffered from depression at various times and was treated for it by a psychiatrist. He was enrolled in the pallidal DBS study protocol 3 years after his last surgery and after also trying the Myobloc trial. At the time of consultation he was taking $12 \mathrm{mg}$ lorazepam, $150 \mathrm{mg}$ amitriptyline, $80 \mathrm{mg}$ propranol$\mathrm{ol}$, and $50 \mathrm{mg}$ rofecoxib daily and smoking medical marijuana to control his painful cervical dystonia.

Examination. On examination he exhibited mild dysarthria and his word finding ability was slow. Results of his general neurological examination were normal, aside from mild tremor with writing and performing the Archimedes spiral. His torticollis was characterized as mild retrocollis with a mild tilt, a moderate twist toward the left, and sagittal shift. Sensory tricks could normalize his neck position and he displayed mild left shoulder elevation as well. Results of swallowing studies were normal preoperatively, although the patient did complain of dry mouth and difficulty swallowing. On neuropsychological assessment mild short-term memory and executive dysfunction were identified, neither of which were severe enough to preclude surgery.
Operation. The patient underwent uneventful bilateral insertion of DBS electrodes in the GPi (Fig. 3), and he experienced an immediate improvement in pain and neck movements postoperatively with electrode placement alone. This benefit wore off 2 weeks later, and programming was initiated with monopolar stimulation of Pole 2 in the left brain and Pole 6 in the right. The parameters of $135 \mathrm{~Hz}, 150-\mu$ sec pulse width, and 2.8 to $3 \mathrm{~V}$ improved his head tremor, neck pain, and movements, although increasing the amplitude to $3.5 \mathrm{~V}$ made his toes curl. Final optimal programming was accomplished using monopolar Electrodes 2 and 6 and settings of $90-\mu$ sec pulse duration, $135 \mathrm{~Hz}$, and $3.4 \mathrm{~V}$.

Postoperative Course. At his 1-year follow-up visit the patient was taking only $200 \mathrm{mg}$ amitriptyline and 120 $\mathrm{mg}$ propranolol a day, having discontinued lorazepam and marijuana. His TWSTRS severity, pain, and disability scores (with maximum scores of 30, 40, and 30, respectively) had dropped from 15,29 , and 19 preoperatively to 4,0 , and 6 postoperatively. His BDI score was 16 preoperatively and 6 at the 1-year follow-up visit (of a total score of 63). The SF-36 quality of life index improved from 82.5 to 124.4 (maximum score in a healthy individual is 146). Neuropsychological and swallowing assessments are pending.

Case 3007

History and Examination. The second patient was a right-handed 48-year-old man who was a sales represen- 

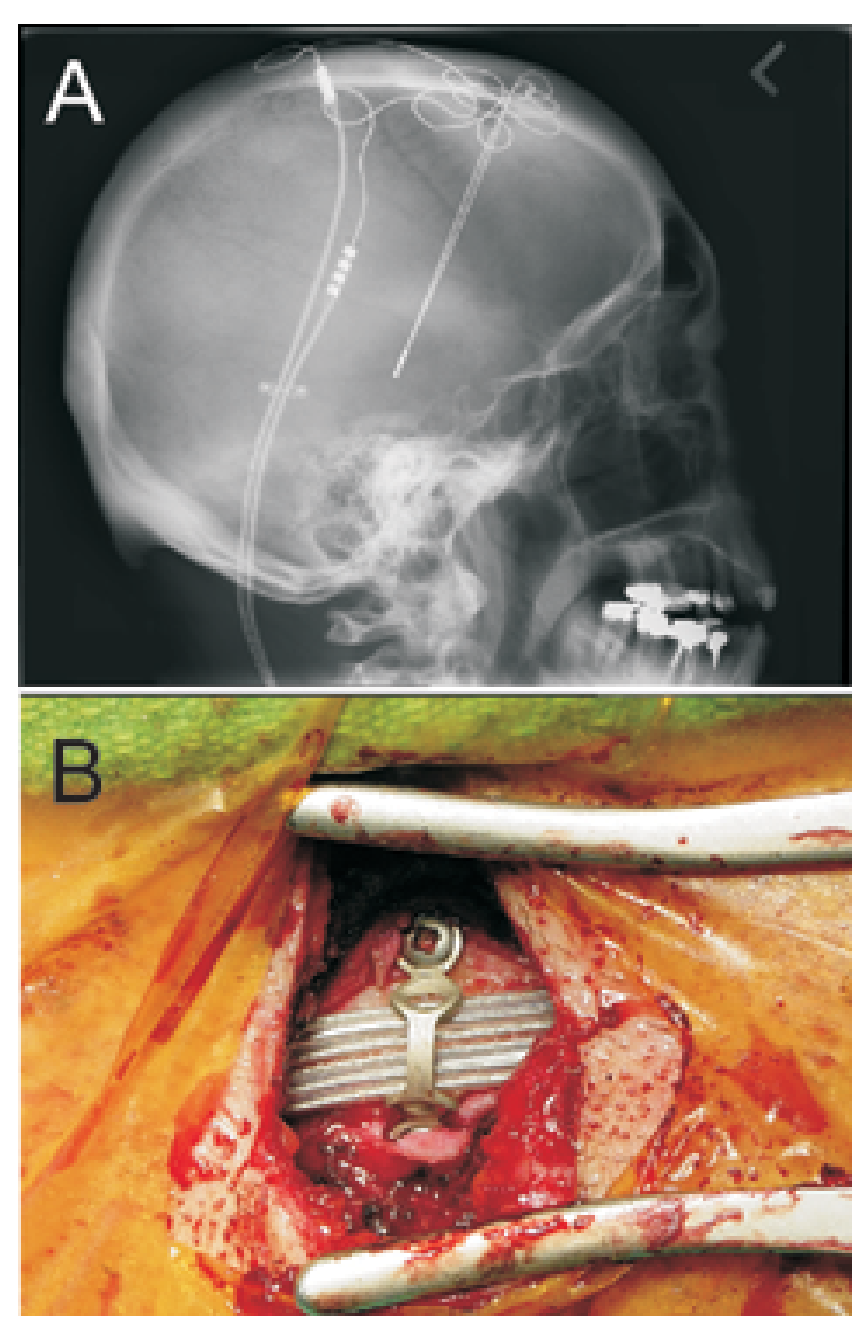

Fig. 2. Illustrations of the method used to minimize hardware complications in patients undergoing DBS for cervical dystonia. A: Lateral skull x-ray film showing the cranial miniplate that is used to prevent the connectors from migrating into the subcutaneous tissues of the neck, where they are more prone to fracture. B: Intraoperative photograph showing the bent miniplate secured to the calvaria, forming a tunnel through which the extension wires pass.

tative and in whom gradual onset of head twist and tilt toward the left developed 5 years before consultation. There was no history of trauma and his family and medical history was unremarkable. No particular sensory trick was useful for relieving his torticollis, aside from pushing his head back onto a hard surface. He was treated with botulinum toxin for the first 3 years, which provided some improvement, but because its effects waned over time he was enrolled in a clinical trial of Myobloc, which did not provide benefit. He continued to work full time, and he did not report significant pain, although he did occasionally take nonsteroidal antiinflammatory drugs. He described the neck movements as the most disabling part of his condition, because his job required so much client contact. He was not depressed (BDI Score 5 of 63). Swallowing studies and neuropsychological testing preoperatively yielded normal to high average results. He did not take any medications. On examination the patient had laterocollis with
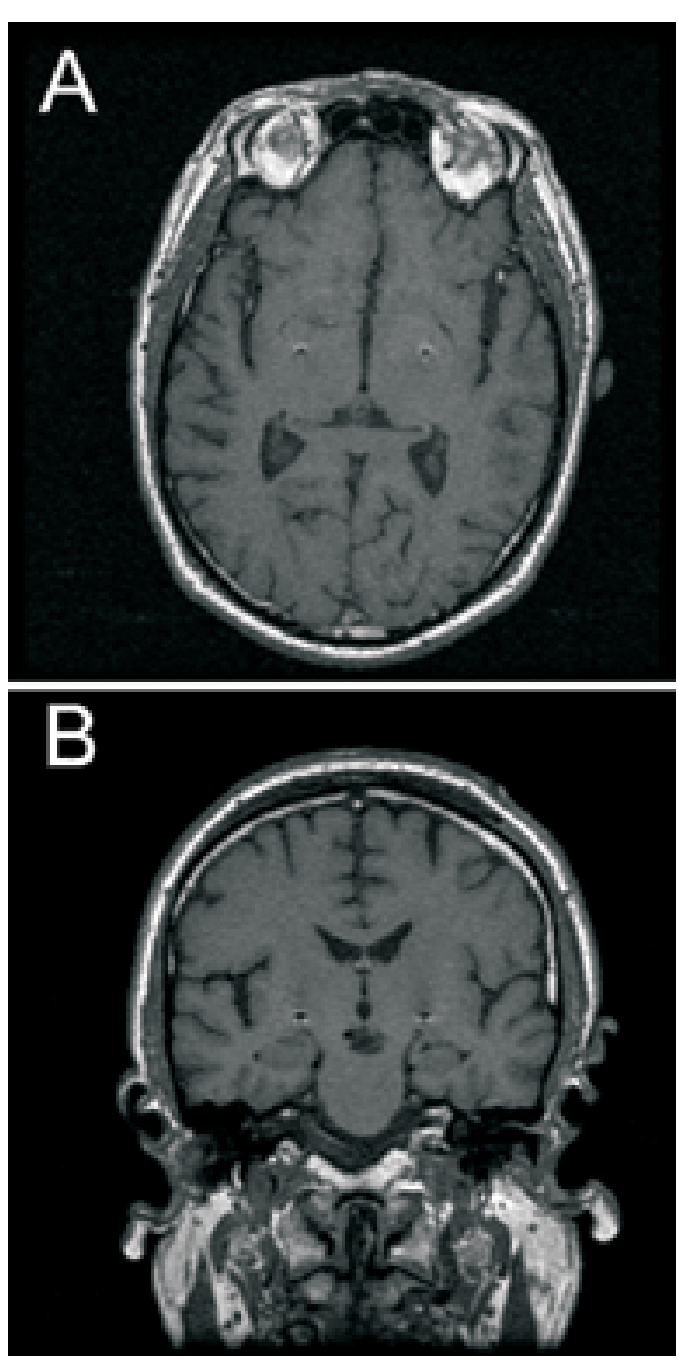

Fig. 3. Postoperative $T_{1}$-weighted $M R$ images demonstrating only the most distal tip of the DBS electrodes in the GPi. A: Axial view. B: Coronal view.

mild rotation to the left and a tilt and prominent elevation of his left shoulder (Video Clip 1).

Click here to view Video Clip 1. Video clip showing the patient in Case 3007 at baseline. First he performs activation/ distracting activities, and then he walks, also displaying the relatively fixed turn and tilt of his neck to the left.

Sensory tricks could only partially relieve the neck movements.

Operation. This patient underwent uncomplicated placement of bilateral electrodes for DBS of the GPi. Electrode location was confirmed on postoperative MR images and an IPG was implanted 4 days later (no immediate benefit was seen). Programming was initiated 1 week later by trying the two lowest electrode poles (150$\mu$ sec pulse width, $135-\mathrm{Hz}$ frequency). Because stimulation at these parameters resulted in paresthesia in the contralateral hand and face, slight speech disturbance, and tightening of the patient's shoulder at amplitudes greater than $2 \mathrm{~V}$, the programming was changed to use the most dorsal poles (3 and 7). 
Postoperative Course. At 3 months the patient was seen for a follow-up visit in the neurosurgery department, and because there was no clinical improvement it was suggested that in reprogramming an attempt should be made to use more ventral electrode poles. At this delayed time point after surgery, bipolar stimulation was initiated using the two lowest poles $(0-/ 1+$ and $4-/ 5+)$ at $180 \mathrm{~Hz}$, with a $180-\mu$ sec pulse width. This allowed an amplitude of $3 \mathrm{~V}$ to be reached without side effects. It resulted in an immediate improvement, within minutes, in subjective tightness of the patient's neck and shoulder muscles, followed by a gradual, visible straightening of his head over the ensuing few days. One week after these settings were initiated the patient returned to the clinic with his posture almost straight. At 6 months postoperatively his sternocleidomastoid muscle remained hypertrophied and his shoulder was slightly elevated, but his TWSTRS severity score had dropped from 14 preoperatively to 6 (Video Clip 2).

Click here to view Video Clip 2. Video clip showing patient in Case 3007 at 6 months postoperatively while performing the same activities as at baseline. There is normalization in his head and neck position, but his left shoulder remains slightly elevated. This video was obtained 3 months after effective programming, as is described in the text.

His pain and disability subscores improved from 21 and 10 before surgery to 4 and 0 , respectively, at his 6-month follow-up visit. His BDI score remained low at 4, and his SF-36 score improved from 95.5 preoperatively to 125.8 at 6 months postoperatively.

\section{Case 3010}

History and Examination. The most recently treated patient was a 62-year-old woman with a 19-year history of neck turning to the right. This occurred after a fall sustained while she was renovating her home. She tried chiropractic manipulations for several years before seeking medical attention. She was enrolled in the initial botulinum toxin trial in 1988, responded quite well, and continued with this treatment for 5 to 6 years until it stopped working. She received trihexyphenidyl, lorazepam, and physical therapy for 6 more years before she enrolled in the Myobloc clinical trial. At the time of enrollment, the patient was taking $6 \mathrm{mg}$ clonazepam, $2.5 \mathrm{mg}$ baclofen, and $1200 \mathrm{mg}$ gabapentin per day, in addition to a nonsteroidal antiinflammatory medication. On examination her torticollis was characterized as rotation to the right without anterocollis, retrocollis, or sagittal shift. She exhibited mild shoulder elevation, and sensory tricks could completely eliminate her abnormal movements.

Operation and Postoperative Course. Surgery was complicated by significant asymmetry in the right and left GP. During the first stereotactic microelectrode exploration, typical pallidal recordings and appropriate microstimulation effects were obtained only in the right brain, not in the left. Therefore, the DBS electrode was implanted only in the right GPi and the patient's return to the operating room 2 weeks later was planned for reexploration and electrode implantation on the left side. The patient suffered shingles in the left V1 territory and therefore surgery on the second side was delayed for 8 weeks to allow the lesions to heal. Stereotactic MR imaging re- vealed evidence of a small, asymptomatic, chronic subdural hematoma on the left side. This was drained through the same burr hole prior to microelectrode exploration. The DBS electrode in the left GPi was implanted after typical recordings and stimulation effects. Three days later both electrodes were connected to the IPG, which placed subcutaneously in the right chest. The postoperative course was complicated by hypoventilation; therefore, her inpatient stay was extended by 4 to 5 days to allow her $\mathrm{O}_{2}$ saturation to remain above $90 \%$.

Programming of the electrodes was initiated within 1 week by using monopolar stimulation (Poles 2 and 7), $210-\mu \mathrm{sec}$ pulse duration, and $180 \mathrm{~Hz}$ at $2 \mathrm{~V}$. One month after programming, her TWSTRS severity, pain, and disability scores had dropped from $18,26.5$, and 11 to 11,0 , and 2 , respectively.

\section{Summary of Preliminary Results}

The total TWSTRS scores improved by 79\%. Figure 4 shows individual subscores: severity subscores had improved significantly at the last follow-up review, from $15.7 \pm 2.1$ to $7.7 \pm 2.9$ (paired t-test, $\mathrm{p}=0.02$ ). Pain and disability subscores improved from $25.5 \pm 4.1$ to $3.3 \pm$ 3.1 (paired t-test, $\mathrm{p}=0.002$ ) and $13.3 \pm 4.9$ to $3.3 \pm 4.2$ (paired t-test $p=0.06$ ), respectively.

\section{DISCUSSION}

Preliminary results in the first three patients treated with DBS of the GPi are encouraging and indicate that cervical dystonia responds favorably to surgery. Although this study is similar to those previously reported, ${ }^{11,17,22,23,31}$ it raises several additional important points. First, rapid clin-

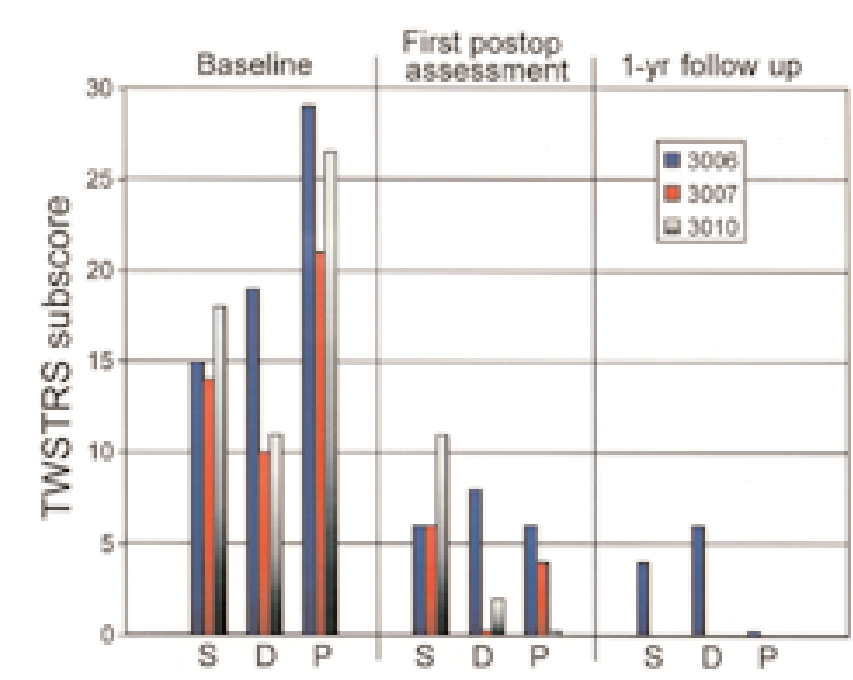

Fig. 4. Bar graph showing the TWSTRS subscores for severity (S), disability (D), and pain (P) before, after, and at 1-year followup review. Each patient is identified by a unique number and color. The patient in Case 3006 is the only one who has reached the 1year follow-up review. The first postoperative assessment was performed at 6 months in the patient in Case 3007 and at 1 month in the patient in Case 3010. Maximum scores are 30, 30, and 40 for severity, disability, and pain, respectively (the lower the score the better the patient outcome). 
ical response was seen in all three patients, in whom an immediate sensation of muscles relaxing was followed within a few days by visible improvement in neck movements. This is contrary to the literature on generalized dystonia; most investigators have reported benefits occurring over several months. ${ }^{5,25,34,35}$ Second, a dissociation has been described; severity of neck movements was not as effectively treated as pain. ${ }^{26}$ We found similar improvements in all subscores of the TWSTRS. Third, the patient in Case 3007 underwent an inadvertent, double-blind test of ineffective followed by effective stimulation. Neither the patient nor the nurse clinician was aware that by using the most dorsal electrodes the GPi was probably not being treated and instead the ventral GPe was affected by the stimulation. The patient frankly admitted that he had given up on DBS helping his cervical dystonia. The treating neurologists had similarly started debating possible reasons why the results in the first patient were so different from those in the second. Nevertheless there is evidence in the literature on PD of the opposite effects of dorsal GPi or ventral GPe stimulation in comparison with ventral DBS of the GPi. ${ }^{21}$ Although we found no worsening of this patient's dystonia with dorsal stimulation, he experienced no clinical change. When his implant was reprogrammed, although he was not expecting any improvement, a dramatic one ensued (Video Clips 1 and 2).

This unusual and inadvertent double-blind test demonstrates that although expectation or placebo effects do exist, ${ }^{8,9}$ they are not likely to be responsible for the benefits seen in this patient. In addition, the importance of electrode placement and programming in the GPi is emphasized. Dystonia is only improved by GPi stimulation, yet abnormal spontaneous firing rates have been reported in both GPi and GPe. ${ }^{37}$ Although the mechanism of efficacy of DBS is uncertain, we can state first that electrode placement is specific and critical, and second that the relatively faster time course for cervical dystonia improvement is different from that observed in generalized dystonia, implying a different mechanism of action.

The multicenter study for which we are reporting preliminary results is presently accruing patients and should be completed within 1 year. Although a limitation of this protocol is the lack of a control group, after polling colleagues in the Canadian Movement Disorders Group, we decided that a control or sham-stimulated group would not be acceptable to the majority of referring neurologists. Efficacy has to be shown first with neurologists performing the severity scoring in a blinded fashion. Adverse events have to be subjected to central review and sham stimulation could only be performed prior to effective programming, because patients would not easily agree to let an effective stimulator program be changed. This contributed to our decision not to propose a doubleblind protocol.

\section{CONCLUSIONS}

Because most studies of DBS for torticollis involve small numbers of patients, a multicenter format was selected to speed patient accrual and to allow our results to be broadly generalized. Because each of the involved centers would likely be performing these surgeries regardless of whether there was a trial underway, our goal was to fol- low these patients prospectively according to the protocol to provide meaningful measures of outcome. Of the five centers involved, three used microelectrode recordings to guide DBS placement and two did not. For generalizability we also did not require a minimum severity score on TWSTRS for a patient to be included in the study. There was a chance that if we had selected patients with the most unresponsive, refractory disease they may have been less likely to respond to any form of treatment. Patients who experience a complication as a result of surgery such that programming cannot be performed (for example, stroke paralysis) will be withdrawn from the study and replaced so that the sample size of 10 patients will be analyzed "as per protocol."'

The final study results will be reported in two ways: results in all patients enrolled as per the "intention-totreat" principle and separately report for the patients treated "as per protocol." All complications will be fully disclosed. If our final results confirm the beneficial effects seen in these first three patients, in further trials we will aim to determine the effects of sham compared with effective stimulation to control for placebo effects, unilateral will be compared with bilateral stimulation, and we will possibly also study surgery compared with best medical management at an earlier stage of cervical dystonia.

\section{Disclaimer}

Devices used in this study were provided by Medtronic Canada; however, the company had no involvement in study design or data analysis.

\section{References}

1. Bereznai B, Steude U, Seelos K, et al: Chronic high-frequency globus pallidus internus stimulation in different types of dystonia: a clinical, video, and MRI report of six patients presenting with segmental, cervical, and generalized dystonia. Mov Disord 17:138-144, 2002

2. Bertrand C, Molina-Negro P, Bouvier G, et al: Observations and analysis of results in 131 cases of spasmodic torticollis after selective denervation. Appl Neurophysiol 50:319-323, 1987

3. Bertrand C, Molina-Negro P, Martinez SN: Combined stereotactic and peripheral surgical approach for spasmodic torticollis. Appl Neurophysiol 41:122-133, 1978

4. Bertrand CM, Lenz FA: Surgical treatment of dystonias, in Tsui JKC, Calne DB (eds): Handbook of Dystonia. New York: Dekker, 1995, pp 329-345

5. Cif L, El Fertit H, Vayssiere N, et al: Treatment of dystonic syndromes by chronic electrical stimulation of the internal globus pallidus. J Neurosurg Sci 47:52-55, 2003

6. Consky ES, Lang AE: Clinical assessments of patients with cervical dystonia, in Jankovic J, Hallett M (eds): Therapy With Botulinum Toxin. New York: Dekker, 1994, pp 211-237

7. Coubes P, Roubertie A, Vayssiere N, et al: Treatment of DYT1generalised dystonia by stimulation of the internal globus pallidus. Lancet 355:2220-2221, 2000

8. de la Fuente-Fernandez R: Uncovering the hidden placebo effect in deep-brain stimulation for Parkinson's disease. Parkinsonism Relat Disord 10:125-127, 2004

9. de la Fuente-Fernandez R, Schulzer M, Stoessl AJ: The placebo effect in neurological disorders. Lancet Neurol 1:85-91, 2002

10. The Deep-Brain Stimulation for Parkinson's Disease Study Group: Deep-brain stimulation of the subthalamic nucleus or the pars interna of the globus pallidus in Parkinson's disease. $\mathbf{N}$ Engl J Med 345:956-963, 2001 
11. Eltahawy HA, Saint-Cyr J, Giladi N, et al: Primary dystonia is more responsive than secondary dystonia to pallidal interventions: outcome after pallidotomy or pallidal deep brain stimulation. Neurosurgery 54:613-621, 2004

12. Friedman AH, Nashold BS Jr, Sharp R, et al: Treatment of spasmodic torticollis with intradural selective rhizotomies. J Neurosurg 78:46-53, 1993

13. Grimes DA: Tremor-easily seen but difficult to describe and treat. Can J Neurol Sci 30 (Suppl 1):S59-S63, 2003

14. Hassler R, Dieckmann G: Stereotactic treatment of different kinds of spasmodic torticollis. Confin Neurol 32:135-143, 1970

15. Hsiung GY, Das SK, Ranawaya R, et al: Long-term efficacy of botulinum toxin A in treatment of various movement disorders over a 10-year period. Mov Disord 17:1288-1293, 2002

16. Hunka K, Suchowersky O, Wood S, et al: Nursing time to program and assess deep brain stimulators in movement disorder patients. J Neurosci Nursing (In Press)

17. Islekel S, Zileli M, Zileli B: Unilateral pallidal stimulation in cervical dystonia. Stereotact Funct Neurosurg 72:248-252, 1999

18. Jahanshahi M, Marion MH, Marsden CD: Natural history of adult-onset idiopathic torticollis. Arch Neurol 47:548-552, 1990

19. Jankovic J, Leder S, Warner D, et al: Cervical dystonia: clinical findings and associated movement disorders. Neurology 41: 1088-1091, 1991

20. Kiss ZHT, Doig K, Eliasziw M, et al: DBS for torticollis: preliminary results from the multicentre Canadian pilot study. Can J Neurol Sci 31 (Suppl 1):S29, 2004 (Abstract)

21. Krack P, Pollak P, Limousin P, et al: Opposite motor effects of pallidal stimulation in Parkinson's disease. Ann Neurol 43: 180-192, 1998

22. Krauss JK, Loher TJ, Pohle T, et al: Pallidal deep brain stimulation in patients with cervical dystonia and severe cervical dyskinesias with cervical myelopathy. J Neurol Neurosurg Psychiatry 72:249-256, 2002

23. Krauss JK, Pohle T, Weber S, et al: Bilateral stimulation of globus pallidus internus for treatment of cervical dystonia. Lancet 354:837-838, 1999

24. Krauss JK, Toups EG, Jankovic J, et al: Symptomatic and functional outcome of surgical treatment of cervical dystonia. J Neurol Neurosurg Psychiatry 63:642-648, 1997

25. Krauss JK, Yianni J, Loher TJ, et al: Deep brain stimulation for dystonia. J Clin Neurophysiol 21:18-30, 2004

26. Kulisevsky J, Lleo A, Gironell A, et al: Bilateral pallidal stimulation for cervical dystonia: dissociated pain and motor improvement. Neurology 55:1754-1755, 2000

27. Loher TJ, Pohle T, Krauss JK: Functional stereotactic surgery for treatment of cervical dystonia: review of the experience from the lesional era. Stereotact Funct Neurosurg 82:1-13, 2004

28. Lozano A, Hutchison W, Kiss Z, et al: Methods for microelectrode-guided posteroventral pallidotomy. J Neurosurg 84: 194-202, 1996

29. Munchau A, Palmer JD, Dressler D, et al: Prospective study of selective peripheral denervation for botulinum-toxin resistant patients with cervical dystonia. Brain 124:769-783, 2001

30. O'Brien C, Brashear A, Cullis P, et al: Cervical dystonia severity scale reliability study. Mov Disord 16:1086-1090, 2001

31. Parkin S, Aziz TZ, Gregory R, et al: Bilateral internal globus pallidus stimulation for the treatment of spasmodic torticollis. Mov Disord 16:489-493, 2001

32. Roubertie A, Echenne B, Cif L, et al: Treatment of early-onset dystonia: update and a new perspective. Childs Nerv Syst 16: 334-340, 2000

33. Schaltenbrand G, Wahren W: Atlas for Stereotaxy of the Human Brain, ed 2. Stuttgart: Thieme, 1977

34. Tronnier VM, Fogel W: Pallidal stimulation for generalized dystonia. Report of three cases. J Neurosurg 92:453-456, 2000

35. Vercueil L, Pollak P, Fraix V, et al: Deep brain stimulation in the treatment of severe dystonia. J Neurol 248:695-700, 2001

36. Vitek JL: Surgery for dystonia, in Bakay RAE (ed): Surgical Treatment of Movement Disorders. Philadelphia: WB Saunders, 1998, Vol 9, pp 345-366

37. Vitek JL, Chockkan V, Zhang JY, et al: Neuronal activity in the basal ganglia in patients with generalized dystonia and hemiballismus. Ann Neurol 46:22-35, 1999

38. Yianni J, Nandi D, Shad A, et al: Increased risk of lead fracture and migration in dystonia compared with other movement disorders following deep brain stimulation. J Clin Neurosci 11: 243-245, 2004

\section{Manuscript received May 27, 2004.}

Accepted in final form June 8, 2004.

Funding for this study was obtained from the Canadian Institutes of Health Research (Dyskinesia and Torticollis Grant) and the Calgary Health Region. Dr. Kiss is a Canadian Institutes of Health Research Clinician-Scientist and a Clinical Investigator for the Alberta Heritage Foundation for Medical Research. Dr. Eliasziw is a Senior Health Scholar for the Alberta Heritage Foundation for Medical Research.

Address reprint requests to: Zelma H. T. Kiss, M.D., Ph.D., Room 182A, HMRB, 3330 Hospital Drive NW, Calgary, Alberta T2N 4N1, Canada. email: zkiss@ucalgary.ca. 